\title{
Superstatistics of Diatomic Molecules with the Shifted Deng-Fan Potential Model
}

\author{
Collins Edet ${ }^{1, *(D)}$, Akpan Ikot ${ }^{1}$ (i) \\ 1 Theoretical Physics Group, Department of Physics, University of Port Harcourt, Nigeria \\ * Correspondence: collinsokonedet@gmail.com (C.E.);
}

Scopus Author ID 57208566600

Received: 31.05.2021; Revised: 10.07.2021; Accepted: 14.07.2021; Published: 13.08.2021

\begin{abstract}
In this article, we discuss the thermodynamic properties of the shifted Deng-Fan potential for $\mathrm{HCl}, \mathrm{CrH}, \mathrm{CuLi}$, and $\mathrm{ScF}$ diatomic molecules using the q-deformed superstatistics approach. The partition function is obtained with the help of the generalized Boltzmann factor from the modified Dirac delta distribution. In addition, thermodynamic functions such as entropy, specific heat capacity, free energy, and mean energy are obtained using the partition function. Our results are presented graphically, and the ordinary statistical quantities are recovered when the deformation parameter tends to zero. Our results may be useful in the study of thermal fluctuations in atomic and molecular systems involving short-range interactions.
\end{abstract}

Keywords: shifted Deng-Fan potential; diatomic molecules; superstatistics.

(C) 2021 by the authors. This article is an open-access article distributed under the terms and conditions of the Creative Commons Attribution (CC BY) license (https://creativecommons.org/licenses/by/4.0/).

\section{Introduction}

In recent times, investigations of diverse molecular potential models have gained the attention of many researchers [1-5]. This is because studies of the rotational motions of diatomic molecules within a given potential are a theme of enormous value in molecular physics[4,5]. In 1929, Philip Morse proposed an empirical potential with three parameters for studying atomic interaction in diatomic molecules[6]. This potential is given as[6];

$$
V(r)=D_{e}\left[1-e^{\alpha\left(r-r_{e}\right)}\right]^{2}
$$

where $D_{e}$ is dissociation energy, $r_{e}$ is the equilibrium internuclear distance, and $\alpha$ is a parameter controlling the width of the potential, well commonly known as the screening parameter $[7,8]$. This progress was a remarkable one in quantum mechanics. It has application in physics and chemistry, particularly in areas of molecular spectroscopy and dynamics[9,10]. Because of its importance in the field of molecular physics[11], the Morse potential has been one of the most useful and convenient models [11,12]. As an empirical potential, this potential gives an exceptional description of the interaction between the two atoms. It is also very realistic to describe the interactions close to the surface [13-16].

In another development, Deng and Fan [17] modified the traditional Morse potential model as an improvement to describe diatomic molecule energy spectra and electromagnetic transitions. This potential reads as follows [17]: 


$$
\begin{aligned}
& V(r)=D_{e}\left(1-\frac{b}{\left(e^{\alpha r}-1\right)}\right)^{2} \\
& b=e^{\alpha r_{e}}-1
\end{aligned}
$$

where $r_{e}$ is the molecular bond length, $D_{e}$ is the dissociation energy, $r$ is the inter-nuclear distance, $\alpha$ the range of the potential well, and $0<r<\infty$. The potential (2) has the correct physical boundary conditions at the origin and infinity, called a generalized Morse potential [17]. The discovery of this potential was another significant move and aroused the interest of several researchers for many years [18-21].

After discovering the highlighted molecular systems, many researchers have modified and or remodeled this potential with more parameters and wider scope of application. The recently developed potentials amongst many others include Manning-Rosen potential [22], Poschl-Teller potential [23], Rosen- Morse potential [24], and Tietz-Wei (STW) oscillator [25]. The aforementioned potentials have been modified and studied [26-28]. These modified and or improved molecular potentials have received remarkable attention. This is due to their various applications in many fields of Physics and Chemistry. Diatomic molecular potentials have been applied to simulate molecular potential energy curves[29], study bound state problems [30-33], predict thermochemical properties of diatomic molecules[34,35], calculations of molecular vibrational partition function [36,37], and prediction of enthalpy and entropy of gaseous dimers[38,39].

It is recognized that thermodynamics is an arm of physics that offers an analysis of macroscopic physical quantities at the molecular level [40]. It employs probability theory to investigate the activities of a system comprised of a large number of particles. The elucidation of the macroscopic theory of thermodynamics in terms of the more abstract microscopic statistical mechanics is one of the most important triumphs of physics in the early twentieth century $[41,42]$.

By way of generalizing thermodynamic studies, Beck and Cohen [43] presented the concept of superstatistics where non-equilibrium systems with complex dynamics in stationary states were considered. Superstatistics is defined as a superposition of two different statistics that describe non-equilibrium systems with a stationary state and parameter fluctuations[43,44]. In non-equilibrium statistical mechanics, $\beta=\frac{1}{k_{\beta} T}$ is not an inverse temperature, thus being seen as a random variable, but regarded as a constant parameter in the case of equilibrium statistical mechanics [4-47].

Much research has been conducted on the concept of superstatistics both in the equilibrium and non-equilibrium statistical mechanics regime [48-52]. Most recently, Sargolzaeipor et al. [46] introduced superstatistics in quantum mechanics and obtained thermodynamic properties of q-deformed Morse potential using this formalism. Okorie et al. [53] discussed the thermodynamic properties of modified Rosen-Morse potential via the qdeformed superstatistics approaches. In another consideration, Sargolzaeipor et al. [54] used superstatistics to study four important physics problems, and the system's thermodynamic properties were evaluated. $q$-deformed superstatistics of the Schrödinger equation in the commutative and non-commutative spaces with the magnetic field was examined by Sargolzaeipor et al. [55]. Sobhani et al. [56] studied the effects of cosmic-string parameters on the thermodynamic properties of a harmonic oscillator within the framework of ordinary 
statistics and the $q$-deformed superstatistics approaches. An innate comprehension of superstatistics of systems involving short-range (exponential-type potential) interactions is required; hence further investigations into the thermodynamics of such systems are still necessary. The aim of this work is to extend the studies carried out by Oyewumi et al. [57] on thermodynamic properties of a shifted Deng-Fan potential to the superstatistics scheme. These results may be useful to studies of thermal fluctuations in atomic and molecular systems involving short-range interactions. It is interesting to note that no study has been carried in this direction with an exponential-type potential.

\section{The Shifted Deng-Fan Oscillator}

Sequel to the discovery of the new generalized Morse potential, Hamzavi et al.[58] proposed an improved expression for the Deng-Fan oscillator potential[17], it was found out that the shifted Deng-Fan was more Morse-like when compared on a plot than the original Deng-Fan oscillator(see. Fig.1 of Ref[58]).

$$
\begin{aligned}
& V(r)=D_{1}\left(1-\frac{b}{\left(e^{\alpha r}-1\right)}\right)^{2}-D_{2} \\
& b=e^{\alpha r_{e}}-1
\end{aligned}
$$

where $D_{e}=D_{1}=D_{2}$. The Morse and the sDF potentials are very close to each other for large values of $r$ in the regions $r \approx r_{e}$ and $r>r_{e}$, but they are very different at $r=0$. Further, if the two potentials are deep $D_{e} \square 1$, they could be well approximated by a harmonic oscillator in the region $r \approx r_{e}$ [58]. This behavior encourages us to apply rotation-vibrational energy level expressions derived in the previous work [57] to calculate the partition function and investigate thermodynamic properties for $\mathrm{HCl}, \mathrm{CrH}, \mathrm{CuLi}$ and $\mathrm{ScF}$ using superstatistical mechanics.

This potential has been studied by some authors. Some of which include; Hamzavi et al. [58], in a maiden consideration, obtained the energy spectra and wave function of the shifted Deng-Fan oscillator potential of a few diatomic molecules using the Nikiforov-Uvarov method [59] by employing Pekeris-type approximation [60] to deal with the centrifugal term. It was noted that their results were found to be in good agreement with those ones previously obtained by the Morse potential. Oyewumi et al. [57] used the Pekeris-type approximation [60] to deal with the centrifugal term. The energy eigenvalues and the shifted Deng-Fan molecular potential wave functions were obtained using the asymptotic iteration method $[62,63]$. Rotational-vibrational energy eigenvalues were presented as follows [57].

$$
E_{n \ell}=\frac{\alpha^{2} \hbar^{2}}{2 m}\left\{\left[\begin{array}{l}
\frac{2 m D_{1}}{\alpha^{2} \hbar^{2}}(b+1)^{2}+ \\
C_{0} \ell(\ell+1)
\end{array}\right]-\left[\frac{\frac{2 m D_{1}}{\alpha^{2} \hbar^{2}}\left[(b+1)^{2}-1\right]+\left(\sqrt{\left(\ell+\frac{1}{2}\right)^{2}+\frac{2 m b^{2} D_{1}}{\alpha^{2} \hbar^{2}}}+\frac{2 n+1}{2}\right)^{2}}{2\left(\sqrt{\left.\left(\ell+\frac{1}{2}\right)^{2}+\frac{2 m b^{2} D_{1}}{\alpha^{2} \hbar^{2}}+\frac{2 n+1}{2}\right)}\right.}\right]^{2}-D_{2}\right.
$$

where $n=0,1,2,3, \ldots, n_{\max }, m$ is the reduced mass of a diatomic molecule, $\hbar$ denotes the reduced Planck constant, $n$ and $\ell$ are the principal and angular momentum quantum numbers, respectively.

Eq.(4) is written in close form as follows; 


$$
\begin{gathered}
E_{n}=\frac{\alpha^{2} \hbar^{2}}{2 m}\left\{A-\left(\frac{B}{2(n+C)}+\frac{n+C}{2}\right)^{2}\right\} \\
A=\frac{2 m}{\alpha^{2} \hbar^{2}}\left[D_{1}(b+1)^{2}-D_{2}\right], B=\frac{2 m D_{1}}{\alpha^{2} \hbar^{2}}\left[(b+1)^{2}-1\right] \\
C=\frac{1}{2}\left[1+\sqrt{1+4\left(\frac{2 m b^{2} D_{1}}{\alpha^{2} \hbar^{2}}\right)}\right]
\end{gathered}
$$

and

Furthermore, thermodynamic properties such as the vibrational mean $U$, specific heat $C$, free energy $F$, and entropy $S$ for the pure vibrational state in the classical limit for these energy eigenvalues were also scrutinized. In the present consideration, we evaluate the thermodynamic quantities of this potential in superstatistics in the next sections. Beck and Cohen[43] have proposed various superstatistics models based on different generalizations of the Boltzmann factor. They also demonstrated that all these models display a universal behavior at low energies or low fluctuations [44]. Consequently, in this paper, we focus only on the modified Dirac delta superstatistics, which is a particular model of superstatistics.

\section{Thermodynamic Functions for Modified Dirac Delta Distribution}

In this section, we introduce the necessary conditions of superstatistics. Superstatistics is a combination of manifold differing statistical models, which scrutinizes the non-linear and non-equilibrium systems. It is noted that this name is chosen because the new statistics represent a kind of "superposition of varying statistics". Superposition of statistical factors can be considered a new attempt to reformulate statistical mechanics [47, 48]. The effective Boltzmann factor of the system can be written as [46, 53]:

$$
B(E)=\int_{0}^{\infty} e^{-\beta^{\prime} E} f\left(\beta^{\prime}, \beta\right) d \beta^{\prime}
$$

where $f\left(\beta^{\prime}, \beta\right)=\delta\left(\beta^{\prime}-\beta\right)$

is a probability density around the global equilibrium inverse temperature $\beta$. For the random variable $\beta^{\prime}$, we assume that the average of these random variables is the same as the global equilibrium inverse temperature, indeed $\left\langle\beta^{\prime}\right\rangle=\beta$. The generalized Boltzmann factor of superstatistics is given by the Laplace transform of the probability density $f\left(\beta^{\prime}, \beta\right)$. There are infinitely many possibilities for choosing the probability density [53].

As a final point, different choices of $f\left(\beta^{\prime}, \beta\right)$ yield various effective Boltzmann factors $B(E)$. Sargolzaeipor et al. [54] elucidated some of the well-known distributions such as uniform, two-level, Gamma, lognormal distribution, and F-distribution.

The generalized Boltzmann factor in q-deformed superstatistics is given as[53]:

$$
B_{q}(E)=e^{-\beta E}\left(1+\frac{q}{2} \beta^{2} E^{2}\right)
$$

where $q$ is the deformation parameter. Details of Eq. (9) can be found in Appendix A of ref.[64] and references therein.

The Superstatistical partition function is written as[46] 


$$
Z=\sum_{n=0}^{\Lambda} B(E)
$$

then, the extension of Eq. (8) is written in q-deformed superstatistic as follows [53]:

$$
f\left(\beta^{\prime}, \beta\right)=a \delta\left(\beta^{\prime}-\beta\right)+b \beta^{\prime} \frac{\partial}{\partial \beta^{\prime}}\left(\delta\left(\beta^{\prime}-\beta\right)\right)+c \beta^{\prime 2} \frac{\partial^{2}}{\partial^{2} \beta^{\prime}} \delta\left(\beta^{\prime}-\beta\right) .
$$

where $a, b$ and $c$ are constants determined by requiring $f\left(\beta^{\prime}, \beta\right)$ to conform to certain physical conditions [46]. Such conditions lead to the values

$$
a=1+q, b=2 q \text { and } c=\frac{1}{2} q
$$

for the constants, where $q$ is a deformation parameter.

The partition function for the modified Dirac delta distribution has the following form [53]:

$$
Z_{q}=\int_{0}^{\infty} B_{q}(E) d n
$$

On substituting eq.(5) into eq.(13), the partition function $Z_{q}$ in $q$-deformed superstatistics for the shifted Deng-Fan potential (3) is given by;

$$
Z_{q}=\int_{0}^{\infty} e^{-\beta\left(\frac{\alpha^{2} \hbar^{2}}{2 m}\left\{A-\left(\frac{B}{2(n+C)}+\frac{n+C}{2}\right)^{2}\right\}\right)}\left(1+\frac{q}{2} \beta^{2}\left(\frac{\alpha^{2} \hbar^{2}}{2 m}\left\{A-\left(\frac{B}{2(n+C)}+\frac{n+C}{2}\right)^{2}\right\}\right)^{2}\right) d n
$$

Eq.(14) is rewritten as ;

$$
Z_{q}=\int_{y_{1}}^{\infty} e^{-\beta\left(\lambda A-\lambda y^{2}\right)}\left(1+\frac{q}{2} \beta^{2}\left(\lambda A-\lambda y^{2}\right)^{2}\right)\left(1+\frac{y}{\sqrt{y^{2}-B}}\right) d y
$$

where $\lambda=\frac{\alpha^{2} \hbar^{2}}{2 m} \quad y=\frac{B}{2(n+C)}+\frac{n+C}{2}$ and $y_{1}=\frac{B}{2 C}+\frac{C}{2}$

The integral in the right-hand side of Eq. (15) is thereafter evaluated with Mathematica software. Thus, we obtain the following expression of the vibrational partition function for a diatomic molecule represented by the shifted Deng-Fan potential energy model,

$$
\begin{aligned}
& Z_{q}=\frac{1}{16(-\beta \lambda)^{5 / 2}} e^{-A \beta \lambda} \beta^{3 / 2} \lambda^{3 / 2} \\
& \sqrt{\beta} \sqrt{\lambda}\left(\begin{array}{l}
-2 e^{y_{1}^{2} \beta \lambda} q \sqrt{-\beta \lambda}\left(\begin{array}{l}
2 y_{1}^{3} \beta \lambda+2 y_{1}^{2} \Omega \beta \lambda- \\
y_{1}(3+4 A \beta \lambda)+\Omega(-3+2(-2 A+B) \beta \lambda)
\end{array}\right)+ \\
\sqrt{\pi}\left(\Sigma+e^{B \beta \lambda} \Xi\right) \\
2 e^{y_{1}^{2} \beta \lambda} \sqrt{-\beta \lambda}\left(\Sigma \text { DawsonF }\left[y_{1} \sqrt{\beta} \sqrt{\lambda}\right]+\Xi D a w \operatorname{son} F[\Omega \sqrt{\beta} \sqrt{\lambda}]\right)
\end{array}\right)-
\end{aligned}
$$

where

$$
\begin{aligned}
& \Xi=8+q(3+4(A-B) \beta \lambda(1+(A-B) \beta \lambda)) \\
& \Sigma=8+q(3+4 A \beta \lambda(1+A \beta \lambda)) \\
& \Omega=\sqrt{-B+y_{1}^{2}}
\end{aligned}
$$


Other thermodynamic functions such as Helmholtz free energy, $F_{S}(\beta)$, entropy, $S_{S}(\beta)$, internal energy, $U_{S}(\beta)$, and specific heat, $C_{S}(\beta)$, functions can be obtained from the partition function(17) with the aid of the following expressions[34]

$$
\begin{aligned}
& F_{S}(\beta)=-\frac{1}{\beta} \ln Z(\beta) \\
& S_{S}(\beta)=-k_{\beta} \frac{\partial F(\beta)}{\partial \beta} \\
& U_{S}(\beta)=-\frac{\partial(\ln Z(\beta))}{\partial \beta} \\
& C_{S}=k_{\beta} \frac{\partial U(\beta)}{\partial \beta}
\end{aligned}
$$

We have obtained the thermodynamic properties via the q-deformed superstatistics approach in terms of two mathematical functions, namely: the Dawson function and the imaginary error function. The Dawson function or Dawson integral in mathematics is denoted as [65]

$$
F(x)=e^{-x^{2}} \int_{0}^{x} e^{y^{2}} d y=\frac{\sqrt{\pi}}{2} e^{-x^{2}} \operatorname{erfi}(x) .
$$

Thus the Dawson's integral is implemented in Mathematica as $\operatorname{Dawson} F(x)$. On the other hand, the imaginary error function is an entire function defined by

$$
\operatorname{Erfi}(x)=i \operatorname{Erf}(i x)
$$

where erf denotes the error function (also called the Gauss error function) is a special function (non-elementary) of sigmoid shape, which occurs in probability, statistics, and partial differential equations. In mathematics, the error function can be denoted as [65]

$$
\operatorname{Erf}(x)=\frac{2}{\sqrt{\pi}} \int_{0}^{x} e^{t^{2}} d t
$$

The imaginary error function is implemented in Mathematica as $\operatorname{Erfi}(x)$.

\section{Results and Discussion}

In our calculations, we have used spectroscopic parameters shown in Table 1 and the conversions: $1 \mathrm{amu}=931.494028 \mathrm{MeV} / \mathrm{c}^{2}, 1 \mathrm{~cm}^{-1}=1.239841875 \times 10^{-4} \mathrm{eV}$ and $\hbar c=1973.29 \mathrm{eV}^{\AA}[30,32]$.

Table 1. Spectroscopic parameters of the molecules used in this work.

\begin{tabular}{c|c|c|c|c} 
& $D_{e}(e V)=D_{1}=D_{2}$ & $r_{e}(\AA)$ & $\alpha\left(\AA^{-\mathbf{1}}\right)$ & $\mu($ a.m.u $)$ \\
\hline $\mathrm{HCl}[57]$ & 4.619031 & 1.2746 & 1.8677 & 0.980105 \\
\hline $\mathrm{CrH}[57]$ & 2.13 & 1.694 & 1.52179 & 0.988976 \\
\hline $\mathrm{CuLi}[57]$ & 1.74 & 2.31 & 1.00818 & 6.259494 \\
\hline $\mathrm{ScF}[57]$ & 5.85 & 1.794 & 1.46102 & 13.35894
\end{tabular}


The variation of the thermodynamic functions with $\beta$ the modified Dirac delta distribution and different diatomic molecules are shown in Figures 1-5. We first obtained a high-temperature partition function and then obtained thermodynamic functions, such as free energy $F(\beta)$, mean vibrational energy $U(\beta)$, specific heat capacity $C(\beta)$, and entropy $S(\beta)$ with the application to some recognized diatomic molecules(for $\mathrm{HCl}, \mathrm{CrH}, \mathrm{CuLi}$ and $S c F)$.

\subsection{Partition function.}

From the variation of the thermodynamic properties obtained, we discovered that in fig. 1, for all the diatomic molecules except $C u L i, Z(\beta)$ monotonically decreases with increasing $\beta$ for $q=0,0.4,0.8$ and 1 . In the case of $C u L i, Z(\beta)$ increases with increasing $\beta$.
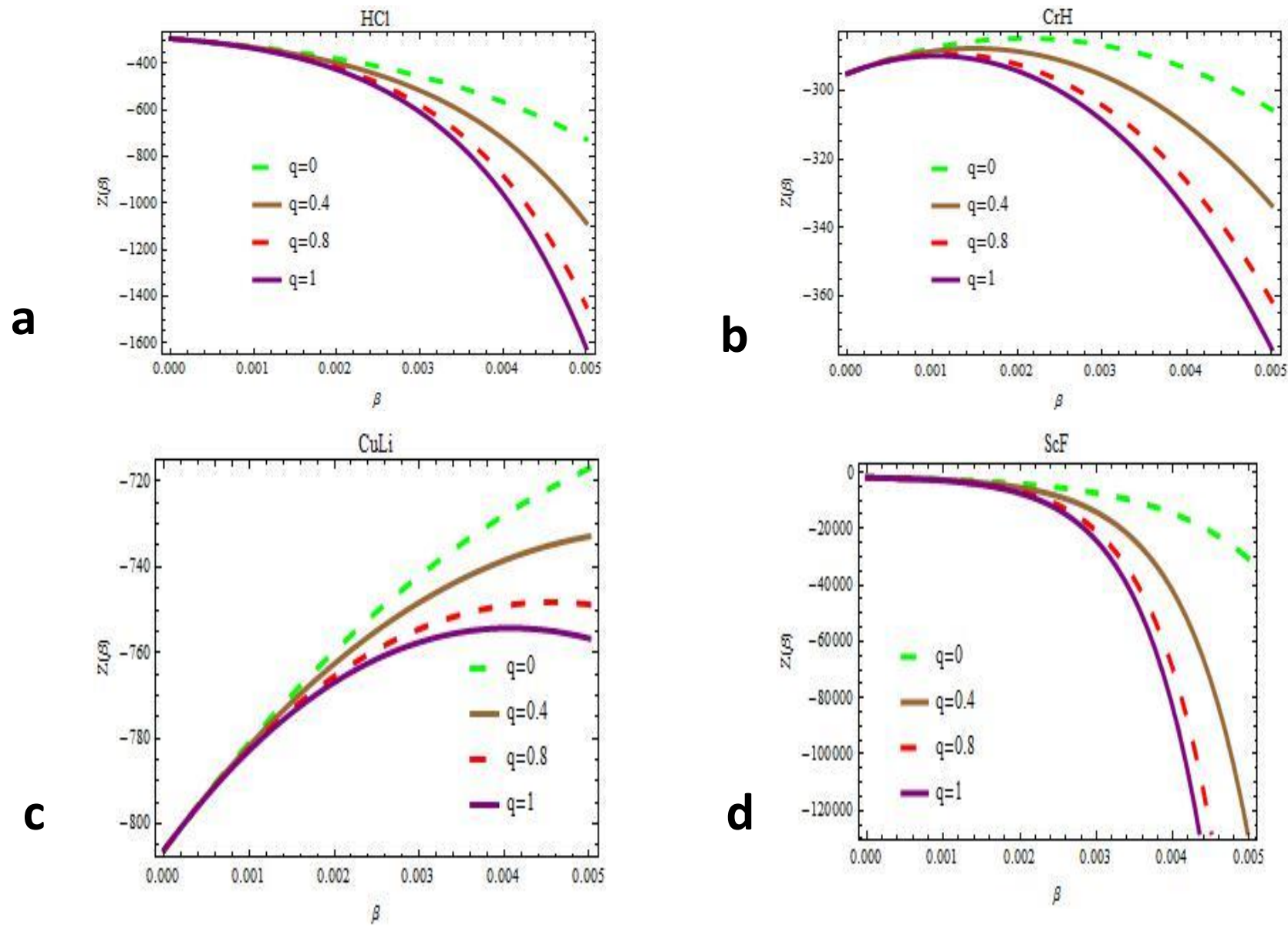

Figure 1. Vibrational partition function $\mathrm{Z}$ of $\mathrm{HCl}, \mathrm{CrH}, \mathrm{CuLi}$ and $S c F$ diatomic molecules as a function of $\beta$ for different $q$.

\subsection{Free energy.}

In Figure 2, we examine the behavior of the mean free energy as it varies in the region $0<\beta<0.08$. It is observed that $F(\beta)$ it decreases monotonically as $\beta$ it increases but increases as the deformation $(q)$ increases for all diatomic molecules considered. 

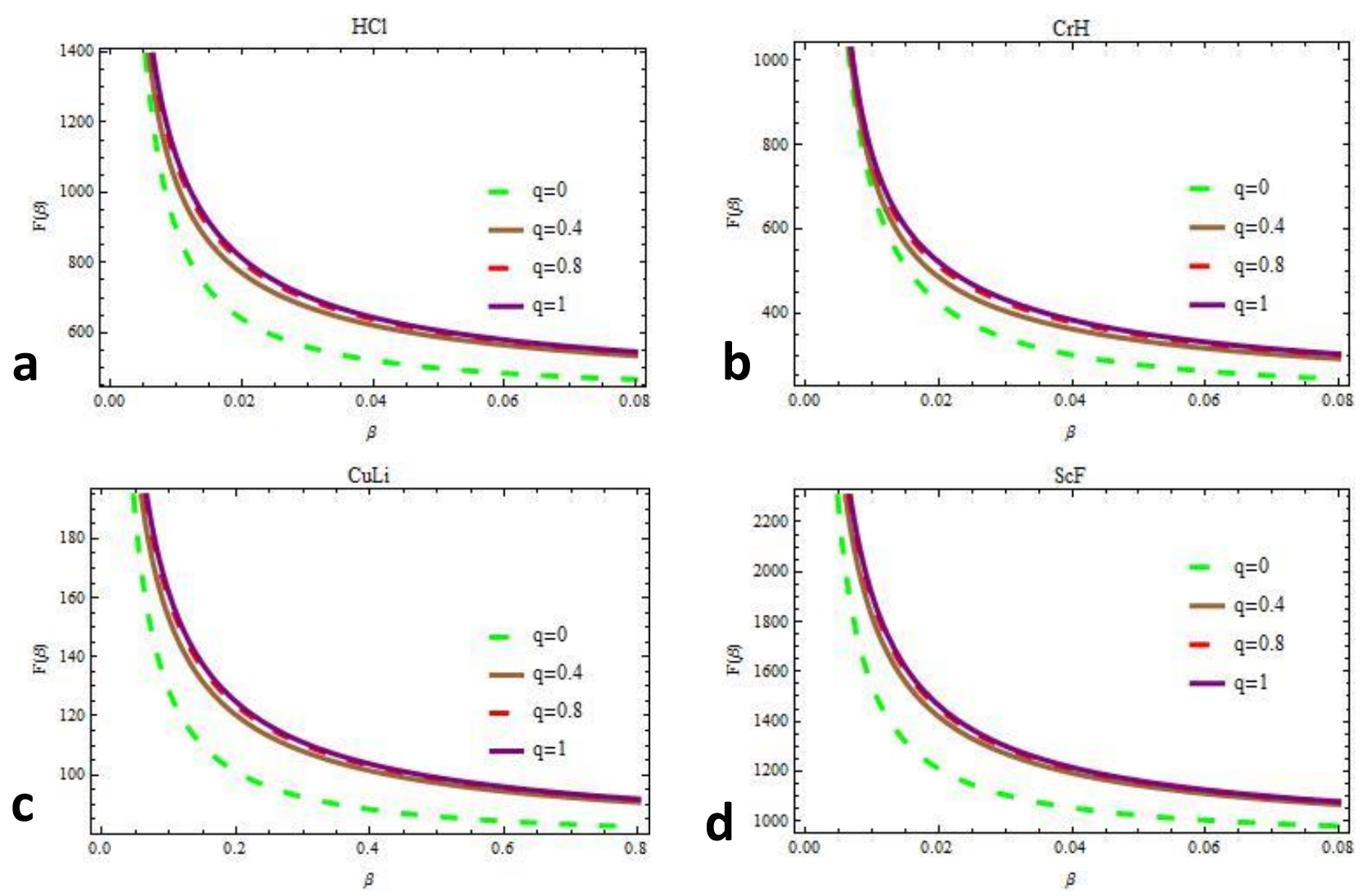

Figure 2. Vibrational mean free energy $F(\beta)$ of $\mathrm{HCl}, \mathrm{CrH}, \mathrm{CuLi}$ and $\mathrm{ScF}$ diatomic molecules as a function of $\beta$ for different $q$.
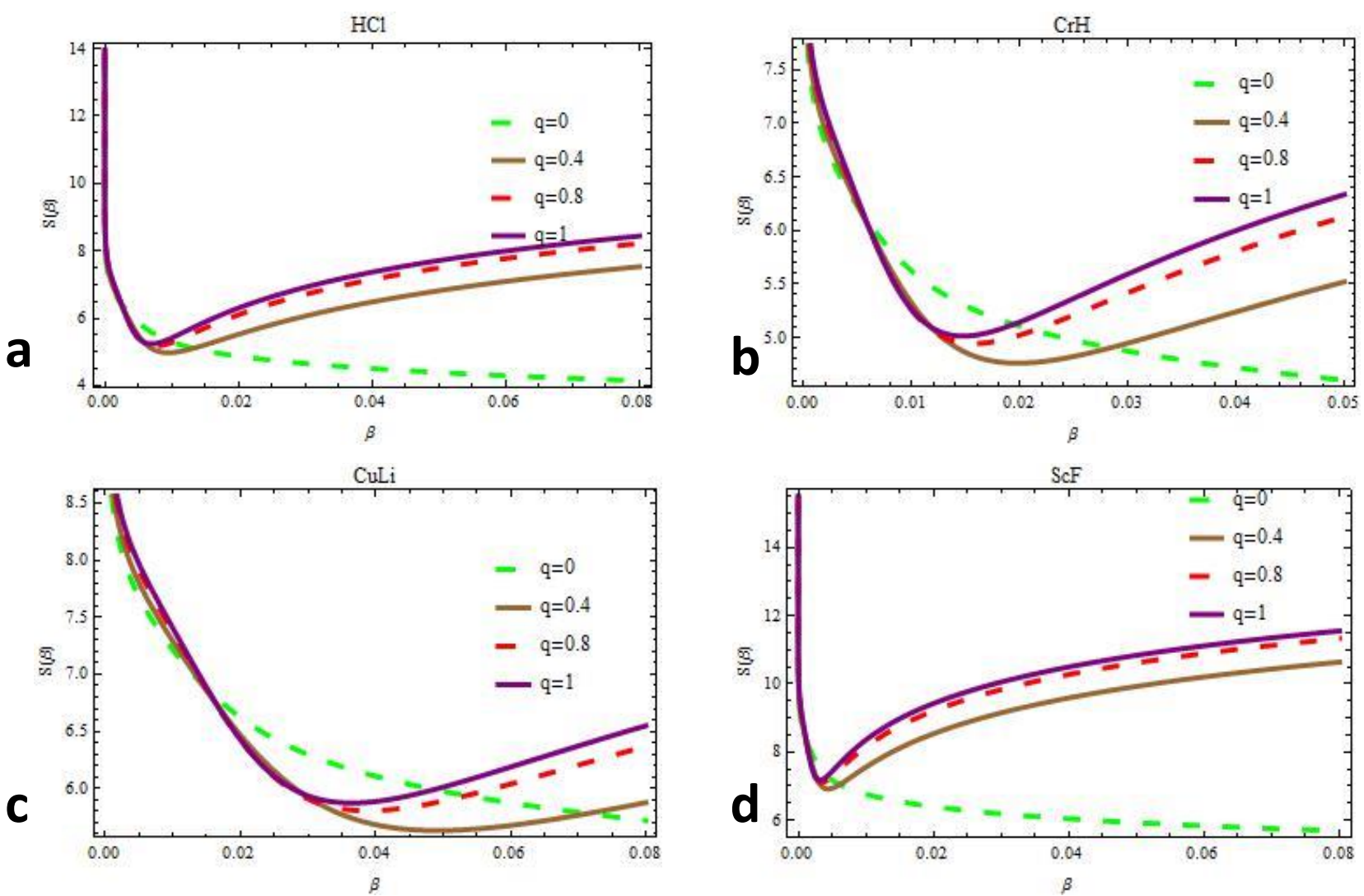

Figure 3. Vibrational entropy $S(\beta)$ of $\mathrm{HCl}, \mathrm{CrH}, \mathrm{CuLi}$ and $\mathrm{ScF}$ diatomic molecules as a function of $\beta$ for different $q$. 


\subsection{Entropy.}

Figure 3 shows the variation of the vibrational entropy $S(\beta)$ of $\mathrm{HCl}, \mathrm{CrH}, \mathrm{CuLi}$ and $S c F$ diatomic molecules as a function of $\beta$ for different $q$. It is clearly revealed that entropy $S(\beta)$ of molecules considered at the start decrease with $\beta$ and lastly increases monotonically with increasing $\beta$ except when $q=0$. We observed a continuous monotonic decrease. The curves for different deformation values show a uniform convergence in the region of low $\beta$ but diverges as $\beta$ increases. More so, it was noted that the entropy of the system increased as the deformation parameter increases. It could be physically said that the deformation increases the disorderliness in the quantum system.

\subsection{Vibrational mean energy.}

Figure 4 explicitly shows the mean vibrational energy $U(\beta)$ of $\mathrm{HCl}, \mathrm{CrH}, \mathrm{CuLi}$ and ScF diatomic molecules as a function of $\beta$ for different $q$. We examined the variation of the mean energy in the region $0<\beta<0.05$. In Fig. 4 , we found that $U(\beta)$ monotonically increases with $\beta$ for $\mathrm{HCl}$ and $\mathrm{ScF}$ but linearly increases with $\beta$ for $\mathrm{CuLi}$ and $\mathrm{CrH}$. However, in Fig. 4, it is also noted that $U(\beta)$ increases with increasing value of the deformation parameter.
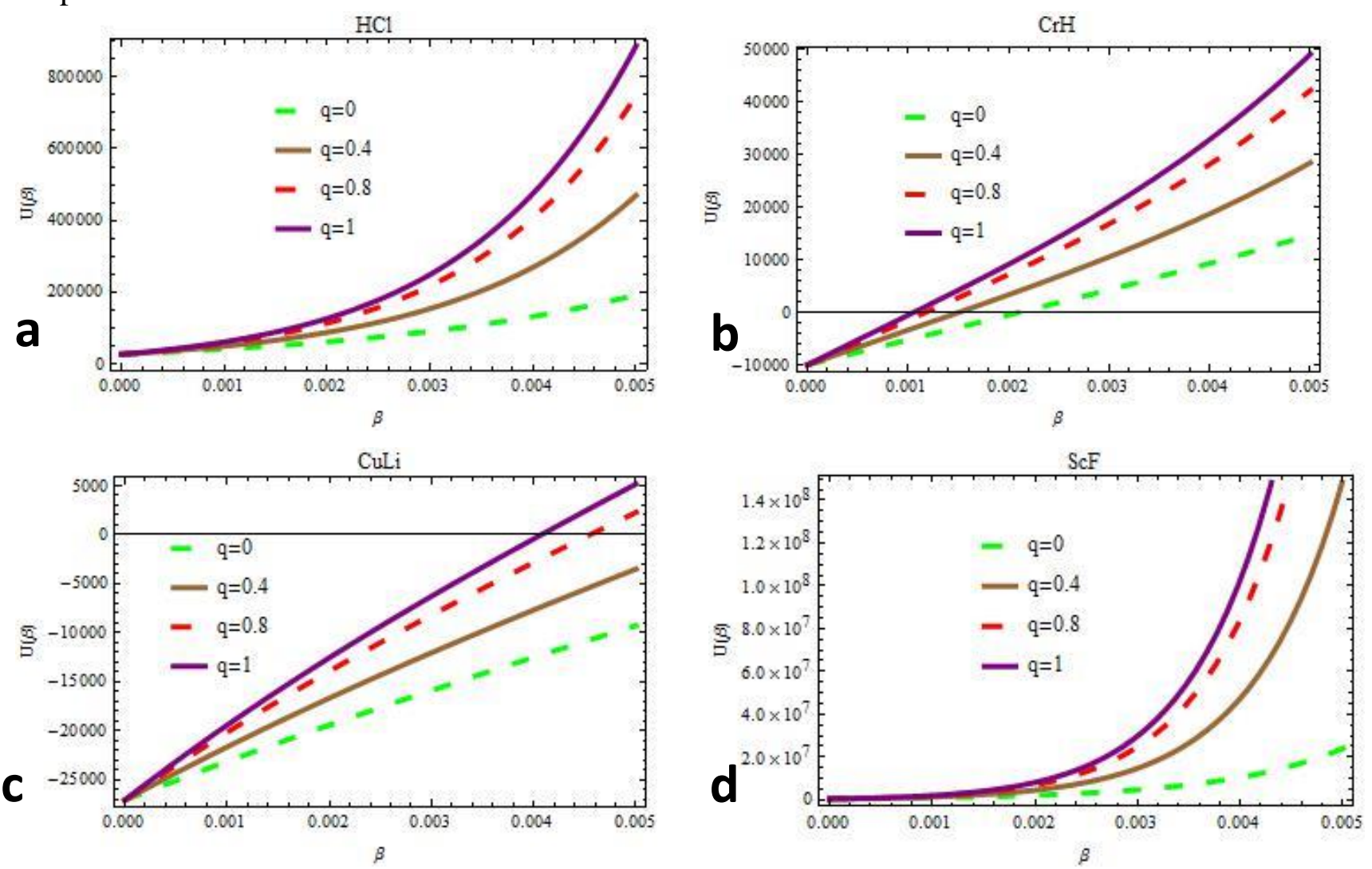

Figure 4: Vibrational mean energy $U(\beta)$ of $\mathrm{HCl}, \mathrm{CrH}, \mathrm{CuLi}$ and $\mathrm{ScF}$ diatomic molecules as a function of $\beta$ for different $q$. 
4.5. Vibrational specific heat capacity.

Figure 5 shows the variation of the vibrational specific heat capacity $C(\beta)$ of $\mathrm{HCl}, \mathrm{CrH}, \mathrm{CuLi}$ and $\mathrm{ScF}$ diatomic molecules as a function of $\beta$ for different $q$. The specific heat increases monotonically with increasing $\beta$ and $q$. We state here that ordinary statistical quantities are recovered when the deformation parameter tends to zero.
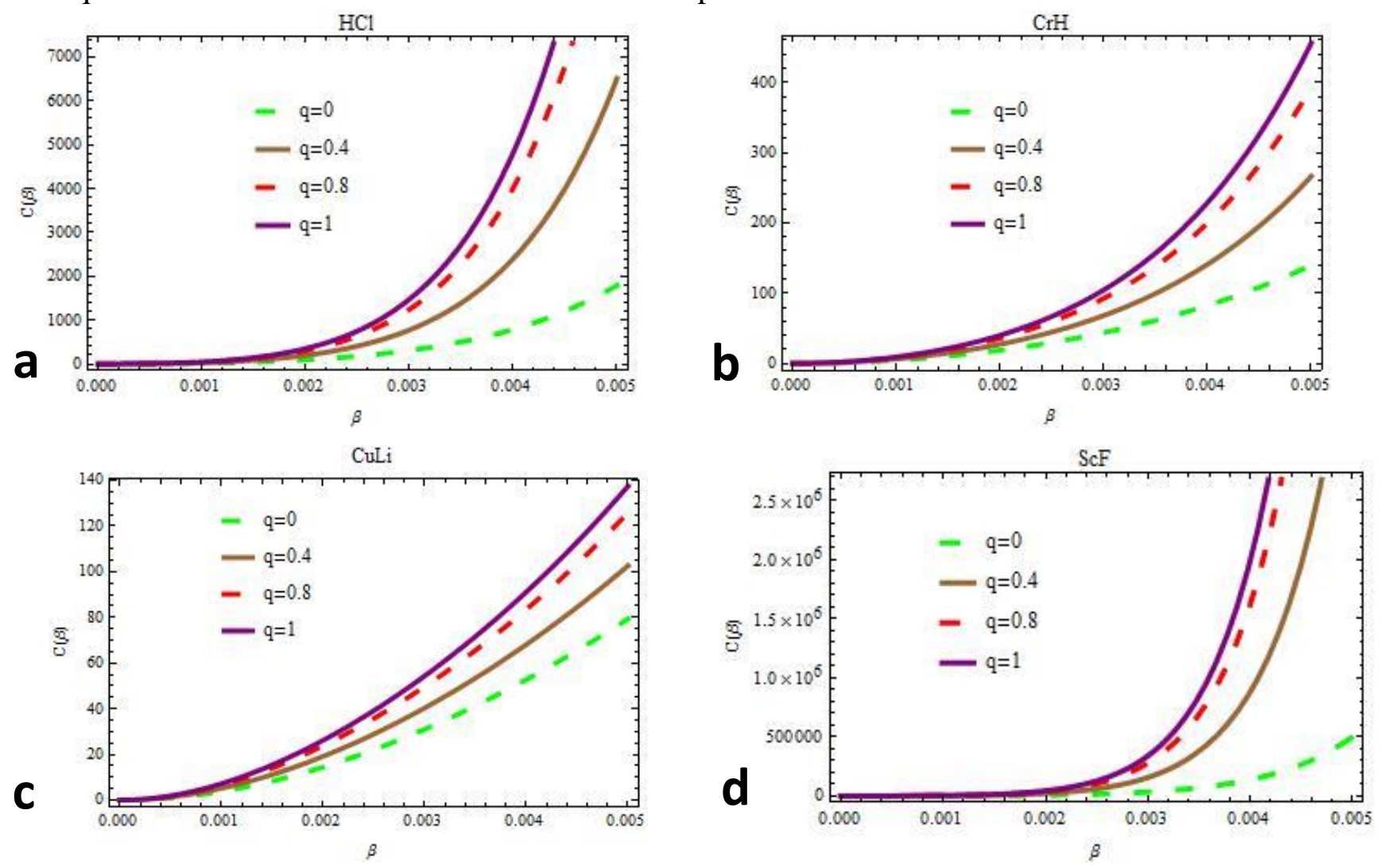

Figure 5: Vibrational specific heat capacity $C(\beta)$ of $\mathrm{HCl}, \mathrm{CrH}, \mathrm{CuLi}$ and $\mathrm{ScF}$ diatomic molecules as a function of $\beta$ for different.

\section{Conclusions}

In this study, the energy spectra obtained for shifted Deng-Fan potential have been adopted to calculate the partition function with the help of the generalized Boltzmann factor for the modified Dirac delta distribution. Other thermodynamic functions such as free energy $F(\beta)$, mean energy $U(\beta)$, entropy $S(\beta)$, and specific heat capacity $C(\beta)$ have been obtained via the superstatistics approach. The variations of these thermodynamic functions with $\beta$ and $q$ in the absence and presence of the deformation parameters have been displayed graphically and discussed extensively for selected diatomic molecules. Specifically, interesting demonstrations are observed in the presence of the deformation parameter. Also, our results agree with the ordinary statistics in the absence of the deformation parameter $(q=0)$.

\section{Funding}

This research received no external funding. 


\section{Acknowledgments}

\section{Collins Edet dedicates this work to his late father (Mr. Okon Edet Udo).}

\section{Conflicts of Interest}

The authors declare no conflict of interest.

\section{References}

1. Khordad, R.; Avazpour, A.; Ghanbari, A. Exact analytical calculations of thermodynamic functions of gaseous substances. Chem. Phys. 2019, 517, 30-35, https://doi.org/10.1016/j.chemphys.2018.09.038.

2. Ocak, Z.; Yanar, H.; Salti, M.; Aydogdu, O. Relativistic spinless energies and thermodynamic properties of sodium dimer molecule. Chem. Phys. 2018, 513, 252-257, https://doi.org/10.1016/j.chemphys.2018.08.015.

3. Liu, HB.; Yi, LZ.; Jia, CS. Solutions of the Klein-Gordon equation with the improved Tietz potential energy model. J. Math. Chem 2018, 56, 2982-2994, https://doi.org/10.1007/s10910-018-0927-0.

4. Edet, C.O.; Okorie, U.S.; Osobonye, G.; Ikot, A.N.; Rampho, G.J.; Sever, R. Thermal properties of DengFan-Eckart potential model using Poisson summation approach. J. Math. Chem. 2020, 58, 989-1013, https://doi.org/10.1007/s10910-020-01107-4.

5. Falaye, B.J.; Ikhdair, S.M.; Hamzavi, M. Spectroscopic study of some diatomic molecules via the proper quantization rule. J. Math. Chem. 2015, 53, 1325-1350, https://doi.org/10.1007/s10910-015-0491-9.

6. Morse, Philip M. "Diatomic molecules according to the wave mechanics. II. Vibrational levels." Phys. Rev. 1929, 34, 57, https://doi.org/10.1103/PhysRev.34.57.

7. Berkdemir, C.; Han, J. Any 1-state solutions of the Morse potential through the Pekeris approximation and Nikiforov-Uvarov method. Chemical physics letters 2005, 409, 203-207, https://doi.org/10.1016/j.cplett.2005.05.021.

8. Edet, C.O.; Okoi, P.O. Any 1-state solutions of the Schrödinger equation for q-deformed Hulthen plus generalized inverse quadratic Yukawa potential in arbitrary dimensions. Revista mexicana de física 2019, 65, 333-344.

9. Jia, C.S.; Zhang, L.H.; Peng, X.L. Improved Pöschl-Teller potential energy model for diatomic molecules. International Journal of Quantum Chemistry 2017, 117, e25383, https://doi.org/10.1002/qua.25383.

10. Jia, C.S.; Diao, Y.F.; Liu, X.J.; Wang, P.Q.; Liu, J.Y.; Zhang, G.D. Equivalence of the Wei potential model and Tietz potential model for diatomic molecules. J. chem. Phys. 2012, 137, 014101, https://doi.org/10.1063/1.4731340.

11. Bag, M.; Panja, M.M.; Dutt, R.; Varshni, Y.P. Modified shifted large-N approach to the Morse oscillator. Phys. Rev. A 1992, 46, 6059, https://doi.org/10.1103/PhysRevA.46.6059.

12. Killingbeck, J.P.; Grosjean, A.; Jolicard, G. The Morse potential with angular momentum. The Journal of Chem. Phys. 2002, 116, 447, https://doi.org/10.1063/1.1418745.

13. Rong, Z.; Kjaergaard, H.G.; Sage, M.L. Comparison of the Morse and Deng-Fan potentials for XH bonds in small molecules. Mol. Phys. 2003., 101, 2285-2294, https://doi.org/10.1080/0026897031000137706.

14. Hu, J.M.; Zhai, J.P.; Wu, F.M.; Tang, Z.K. Molecular dynamics study of the structures and dynamics of the iodine molecules confined in AlPO4-11 crystals. J. Phys. Chem. B 2010, 114, 16481-16486, https://doi.org/10.1021/jp1076615.

15. Inci, I.; Bonatsos, D.; Boztosun, I. Electric quadrupole transitions of the Bohr Hamiltonian with the Morse potential. Physical Review C 2011, 84, 024309, https://doi.org/10.1103/PhysRevC.84.024309.

16. Zhang, P., Long, HC. \& Jia, CS. Solutions of the Dirac equation with the Morse potential energy model in higher spatial dimensions. Eur. Phys. J. Plus 2016, 131, 117, https://doi.org/10.1140/epjp/i2016-16117-4.

17. Deng, Z.H.; Fan, Y.P. A potential function of diatomic molecules. J. Shandong Uni. (Natural Science) 1957, 1 .

18. Zhang, L.H.; Li, X.P.; Jia, C.S. Approximate solutions of the Schrödinger equation with the generalized Morse potential model including the centrifugal term. Intl. J. Quant. Chem. 2011, 111, 1870-1878, https://doi.org/10.1002/qua.22477.

19. Dong, S.H.; Gu, X.Y. Arbitrary 1 state solutions of the Schrödinger equation with the Deng-Fan molecular potential. J. Phys.: Conf. Series 2008, 96, 012109, https://doi.org/10.1088/1742-6596/96/1/012109. 
20. Lucha, W.; Schöberl, F.F. Solving the Schrödinger equation for bound states with Mathematica 3.0. Intl. J. Mod. Phys. C 1999, 10, 607-619, https://doi.org/10.1142/S0129183199000450.

21. Rong, Z.; Kjaergaard, H.G.; Sage, M.L. Comparison of the Morse and Deng-Fan potentials for XH bonds in small molecules. Mol. Phys. 2003, 101, 2285-2294, https://doi.org/10.1080/0026897031000137706.

22. Manning, M.F.; Rosen, N. A potential function for the vibrations of diatomic molecules. Phys. Rev. 1933, 44 , 951-954.

23. Pöschl, G.; Teller, E. Bemerkungen zur Quantenmechanik des anharmonischen Oszillators. Z. Physik 1933, 83, 143-151. https://doi.org/10.1007/BF01331132.

24. Rosen, N.; Morse, P.M. On the vibrations of polyatomic molecules. Phys. Rev. 1932, 42, 210, https://doi.org/10.1103/PhysRev.42.210.

25. Tietz, T. Potential-energy function for diatomic molecules. J. Chem. Phys. 1963, 38, 3036, https://doi.org/10.1063/1.1733648.

26. Sun, G.H.; Dong, S.H. Relativistic treatment of spinless particles subject to a Tietz-Wei oscillator. Commun. Theor. Phys. 2012, 58, 195. https://doi.org/10.1088/0253-6102/58/2/04.

27. Wang, P.Q.; Liu, J.Y.; Zhang, L.H.; Cao, S.Y.; Jia, C.S. Improved expressions for the Schiöberg potential energy models for diatomic molecules. J. Mol. Spect. 2012, 278, 23-26, https://doi.org/10.1016/j.jms.2012.07.001.

28. Jia, C.S.; Zhang, L.H.; Wang, C.W. Thermodynamic properties for the lithium dimer. Chem. Phys. Lett. 2017, 667, 211-215, https://doi.org/10.1016/j.cplett.2016.11.059.

29. Fu, K.X.; Wang, M.; Jia, C.S. Improved five-parameter exponential-type potential energy model for diatomic molecules. Commun. Theor. Phys. 2019, 71, 103, https://doi.org/10.1088/0253-6102/71/1/103.

30. Edet, C.O.; Okorie, K.O.; Louis, H.; Nzeata-Ibe, N.A. Any $l$-state solutions of the Schrodinger equation interacting with Hellmann-Kratzer potential model. Indian $J$ Phys. 2020, 94, 243-251 https://doi.org/10.1007/s12648-019-01467-X.

31. Edet, C.O.; Okorie, U.S.; Ngiangia, A.T.; Ikot, A.N. Bound state solutions of the Schrodinger equation for the modified Kratzer potential plus screened Coulomb potential. Indian J Phys. 2020, 94, 425-433 https://doi.org/10.1007/s12648-019-01477-9.

32. Okorie, U.S.; Ikot, A.N.; Edet, C.O.; Akpan, I.O.; Sever, R.; Rampho, G.J. Solutions of the Klein Gordon equation with generalized hyperbolic potential in D-dimensions. J. Phys. Commun. 2019, 3, 095015, https://doi.org/10.1088/2399-6528/ab42c6.

33. Edet, C.O.; Okoi, P.O.; Chima, S.O. Analytic solutions of the Schrödinger equation with non-central generalized inverse quadratic Yukawa potential. Rev. Bras. Ens. Fís. 2020, 42, http://dx.doi.org/10.1590/1806-9126-RBEF-2019-0083.

34. Edet, C.O.; Ikot, A.N. Effects of Topological Defect on the Energy Spectra and Thermo-magnetic Properties of CO Diatomic Molecule. J Low Temp Phys. 2021, 203, 84-111, https://doi.org/10.1007/s10909-021-025779.

35. Rampho, G.J.; Ikot, A.N.; Edet, C.O.; Okorie, U. S. Energy spectra and thermal properties of diatomic molecules in the presence of magnetic and AB fields with improved Kratzer potential, Mol. Phys. 2021, 119, e1821922, https://doi.org/10.1080/00268976.2020.1821922.

36. Okorie, U.S.; Edet, C.O.; Ikot, A.N.; Rampho, G.J.; Sever, R. Thermodynamic functions for diatomic molecules with modified Kratzer plus screened Coulomb potential. Indian J Phys. 2021, 95, 411-421 https://doi.org/10.1007/s12648-019-01670-w.

37. Wang, C.W.; Wang, J.; Liu, Y.S.; Li, J.; Peng, X.L.; Jia, C.S.; Zhang, L.H.; Yi, L.Z.; Liu, J.Y.; Li, C.J.; Jia, X. Prediction of the ideal-gas thermodynamic properties for water. J. Mol. Liq. 2021, 321, 114912, https://doi.org/10.1016/j.molliq.2020.114912.

38. Oluwadare, O.J.; Oyewumi, K.J.; Abiola, T.O. Thermodynamic properties of some diatomic molecules confined by an harmonic oscillating system. Indian J Phys 2021, https://doi.org/10.1007/s12648-021-021395 .

39. Horchani, R., Shafii, S.A., Friha, H.; Jelassi, H. A Straightforward Model for Molar Enthalpy Prediction of $\mathrm{CsO}, \mathrm{CsF}$, and $\mathrm{CsCl}$ Molecules Via Shifted Tietz-Wei Potential. Int J Thermophys. 2021, $42,84$. https://doi.org/10.1007/s10765-021-02839-4.

40. Ebeling, W.; Sokolov, M.I. Statistical Thermodynamics and Stochastic Theory of Nonequilibrium Systems, World Scientific, Singapore 2005 pp. 312.

41. Landsberg, P.T. Thermodynamics and Statistical Mechanics, Dover, New York 1991. 
42. Edet, C. O.; Ikot, A N. Analysis of the impact of external fields on the energy spectra and thermo-magnetic properties of $\mathrm{N}_{2}, \mathrm{I}_{2}, \mathrm{CO}, \mathrm{NO}$ and $\mathrm{HCl}$ diatomic molecules, Mol. Phys. 2021, https://doi.org/10.1080/00268976.2021.1957170.

43. Beck, C.; Cohen, E.G.D. Superstatistics. Physica A 2003, 322, 267-275, https://doi.org/10.1016/S03784371(03)00019-0.

44. Beck, C.; Cohen, E.G.D. Superstatistical generalization of the work fluctuation theorem. Physica A 2004, 344, 393-402, https://doi.org/10.1016/j.physa.2004.06.001.

45. Wilk, G.; Włodarczyk, Z. Interpretation of the nonextensivity parameter q in some applications of Tsallis statistics and Lévy distributions. Physical Review Lett. 2000, 84, 2770, https://doi.org/10.1103/PhysRevLett.84.2770.

46. Hassanabadi, H.; Sargolzaeipor, S.; Chung, W.S. Superstatistics properties of q-deformed Morse potential in one dimension. Physica A 2018, 508, 740-747, https://doi.org/10.1016/j.physa.2018.05.125.

47. Beck, C. Generalized statistical mechanics of cosmic rays. Physica A 2004, 331, 173-181, https://doi.org/10.1016/j.physa.2003.09.025.

48. Beck, C. Superstatistics: theory and applications. Continuum Mech. Thermodyn. 2004, 16, $293-304$ (2004). https://doi.org/10.1007/s00161-003-0145-1

49. Edet, C.O.; Amadi, P.O.; Okorie, U.S.; Tas, A.; Ikot, A.N.; Rampho, G. Solutions of Schrodinger equation and thermal properties of generalized trigonometric Poschl-Teller potential. Rev. Mex. Fís. 2020, 66, 824839.

50. Tsallis, C.; Souza, A.M. Constructing a statistical mechanics for Beck-Cohen superstatistics. Phys. Rev. E 2003, 67, 026106. https://doi.org/10.1103/PhysRevE.67.026106

51. Beck, C. Lagrangian acceleration statistics in turbulent flows. EPL (Europhysics Letters) 2003, 64, 151, https://doi.org/10.1209/epl/i2003-00498-4.

52. Reynolds, A.M. Superstatistical mechanics of tracer-particle motions in turbulence. Phys. Rev. lett. 2003, 91 , 084503, https://doi.org/10.1103/PhysRevLett.91.084503.

53. Okorie, U.S.; Ikot, A.N.; Rampho, G.J.; Sever, R. Superstatistics of modified Rosen-Morse potential with Dirac delta and uniform distributions. Commun. Theor. Phys. 2019, 71, 1246, https://doi.org/10.1088/0253$6102 / 71 / 10 / 1246$.

54. Sargolzaeipor, S.; Hassanabadi, H.; Chung, W.S. Superstatistics with different kinds of distributions in the deformed formalism. Eur. Phys. J. Plus 2018, 133, 125, https://doi.org/10.1140/epjp/i2018-11962-7.

55. Sargolzaeipor, S., Hassanabadi, H. \& Chung, W.S. q-deformed superstatistics of the Schrödinger equation in commutative and non-commutative spaces with magnetic field. Eur. Phys. J. Plus 2018, 133, 5, https://doi.org/10.1140/epjp/i2018-11827-1.

56. Sobhani, H.; Hassanabadi, H.; Chung, W.S. Effects of cosmic-string framework on the thermodynamical properties of anharmonic oscillator using the ordinary statistics and the $q$-deformed superstatistics approaches. Eur. Phys. J. C 2018, 78, 106, https://doi.org/10.1140/epjc/s10052-018-5581-y.

57. Oyewumi, K.J.; Falaye, B.J.; Onate, C.A.; Oluwadare, O.J.; Yahya, W.A. Thermodynamic properties and the approximate solutions of the Schrödinger equation with the shifted Deng-Fan potential model. Mol. Phys. 2014, 112, 127-141. https://doi.org/10.1080/00268976.2013.804960.

58. Hamzavi, M.; Ikhdair, S.M.; Thylwe, K.E. Equivalence of the empirical shifted Deng-Fan oscillator potential for diatomic molecules. J Math Chem 2013, 51, 227-238, https://doi.org/10.1007/s10910-012-0075-x.

59. Nikiforov, A.F.; Uvarov, V.B. Special Functions of Mathematical Physics (ed.) A Jaffe 1998.

60. Greene, R.L.; Aldrich, C. Variational wave functions for a screened Coulomb potential. Phys. Rev. A 1976, 14, 2363, https://doi.org/10.1103/PhysRevA.14.2363.

61. Chen, T.; Diao, Y.F.; Jia, C.S. Bound state solutions of the Klein-Gordon equation with the generalized Pöschl-Teller potential. Physica Scripta 2009, 79, 065014, https://doi.org/10.1088/0031-8949/79/06/065014.

62. Ciftci, H.; Hall, R.L.; Saad, N. Asymptotic iteration method for eigenvalue problems. J. Phys. A: Math. Gen. 2003, 36, 11807, https://doi.org/10.1088/0305-4470/36/47/008.

63. Ikot, A.N.; Edet, C.O.; Okorie, U.S.; Abdel-Aty, A.H.; Ramantswana, M.; Rampho, G.J.; Alshehri, N.A.; Elagan, S.K.; Kaya, S. Solutions of the 2D Schrodinger equation and its thermal properties for improved ultra-generalized exponential hyperbolic potential (IUGE-HP). Eur. Phys. J. Plus 2021, 136, 434, https://doi.org/10.1140/epjp/s13360-021-01408-w.

64. Sargolzaeipor, S.; Hassanabadi, H.; Chung, W.S. Superstatistics of two electrons quantum dot. Mod. Phys. Lett. A 2019, 34, 1950023, https://doi.org/10.1142/S0217732319500238. 
65. Abramowitz, M.; Stegun, I.A. (Eds.). Handbook of mathematical functions with formulas, graphs, and mathematical tables (Vol. 55). US Government printing office 1964. 